\title{
Design and Sizing on a Parametric Structural Model for a UCAV Configuration for Loads and Aeroelastic Analysis
}

\author{
Arne Voss • Thomas Klimmek
}

Received: date / Accepted: date

\begin{abstract}
The authors present the setup of a parametric structural finite element model for the loads and aeroelastic analysis of an unmanned combat air vehicle (UCAV). The DLR-F19 is a "flying wing" configuration with a geometry based on previous research conducted in the scope of the "Mephisto" project and its predecessors "FaUSST" and "UCAV2010". While a considerable body of knowledge exists regarding conventional configurations, unconventional configurations lack that same level of experience, and data for comparison is rarely available. Using an adequate structural model, the conceptual design stage becomes more sophisticated and already allows for the investigation of physical effects at an early stage of the design process. Strategies for structural modeling and proper condensation, aero-structural coupling, loads integration, control surface attachment, and the use of composite materials are addressed in this paper. The resulting model is sized for minimum structural weight, taking into account 216 load cases. In addition, a comprehensive loads analysis campaign is conducted and the resulting loads are evaluated at defined monitoring stations. In addition to maneuver loads, quasi-static gust loads are calculated using the Pratt formula and compared to results obtained from a dynamic 1-cosine gust simulation. The reasons
\end{abstract}

\section{Arne Voss}

DLR - German Aerospace Center

Institute of Aeroelasticity

Bunsenstrasse 10

D-37073 Göttingen

Tel.: +49-551-709-2514

E-mail: arne.voss@dlr.de

Thomas Klimmek

DLR - German Aerospace Center

Institute of Aeroelasticity for higher loads of the Pratt formula based method are discussed. The conclusion is that the Pratt formula is suitable for the preliminary sizing of "flying wing" configurations.

Keywords UCAV · parametrized structural modeling $\cdot$ loads $\cdot$ sizing

\section{Introduction}

The DLR-F19 is a "flying wing" configuration for an unmanned combat air vehicle (UCAV). The geometry is based on previous research conducted in the scope of the DLR project "Mephisto" and its predecessors "FaUSST" and "UCAV2010". It was used for wind tunnel investigations (e.g. [13,14,32]) and real-size design concepts (e.g. $[18,20])$. While there is a considerable amount of knowledge about conventional configurations, there is little experience regarding unconventional configurations and data for comparison is rarely available. Comparable UCAVs are the Northrop Grumman X47B [3,4] or Boeing/Nasa X-48 [1,2], both experimental demonstrators, while the Northrop B-2 $[5,33]$ is a much larger stealth bomber. On the civil side and for lower speed, are the sailplanes SB 13 from Akaflieg Braunschweig [27] and the AK-X prototype from Akaflieg Karlsruhe [8].

Through the use of an adequate structural model, the conceptual design becomes more sophisticated by enabling detailed loads and aeroelastic analysis such those involving maneuvering loads, gust encounters or flutter behavior. A comprehensive and detailed level and the use of parametrization allow for the investigation of physical effects at an early stage of the design process. The importance of such a process is underlined 
e.g. by companies such as Boeing [6] or Lockheed Martin [7]. Similar processes are developed for wing mass estimations e.g. within Airbus [15,31]. Other work concentrates on multidisciplinary design and on achieving a feasible and flyable configuration $[22,28,34]$. This paper focuses on the challenges imposed by the geometry of the DLR-F19 being significantly different in some aspects from that of classical aircraft. All of this requires different and/or new modeling strategies. The aircraft is much more compact and integrated, so that e.g. established aero-structural coupling methods need to be enhanced. The loads evaluation also gets more complicated. The use of a detailed structural model instead of a condensed one for loads calculation significantly increases computational requirements but also leads to a more realistic loads distribution, especially in the chordwise direction. Furthermore, investigations on the control surface loads are more realistic. Depending on stability margins, control surface loads are presumably high, as control surfaces for level flight conditions are attached directly to the wing resulting in rather short moment arms.

The DLR parametric modeling process is outlined in Section 2. The resulting aeroelastic model is presented in Section 3 with a discussion of the differences and challenges compared to a classical configuration. Once the model is set up, a sizing loop is started to minimize the structural weight of the aircraft. The inputs and results are discussed in Section 4. In Section 5, a comprehensive loads analysis is conducted to identify the critical load cases for the DLR-F19 configuration. In addition, dynamic 1-cosine gust calculations are performed and compared to results from the Pratt formula. The latter is used to account for gust loads in the sizing loop.

\section{Parametric Modeling, Loads and Design Process}

To generate such a parametric model, the in-house software ModGen [17] is used. ModGen is a parametrized processor to set up MSC Nastran finite element models as well as aerodynamic models, optimization models for structural sizing, and other MSC Nastran simulation models (e.g. for mass modeling). The input for this process is basic information such as profile data, geometrical dimensions and design parameters of the wing box (e.g. number, position and orientation of spars, ribs and stringer). The software has various modules that generate the individual aircraft components depicted in Fig. 1 and creates nearly all data required for various MSC Nastran calculations, depending on the selected MSC Nastran solution. In this way, parameters can be

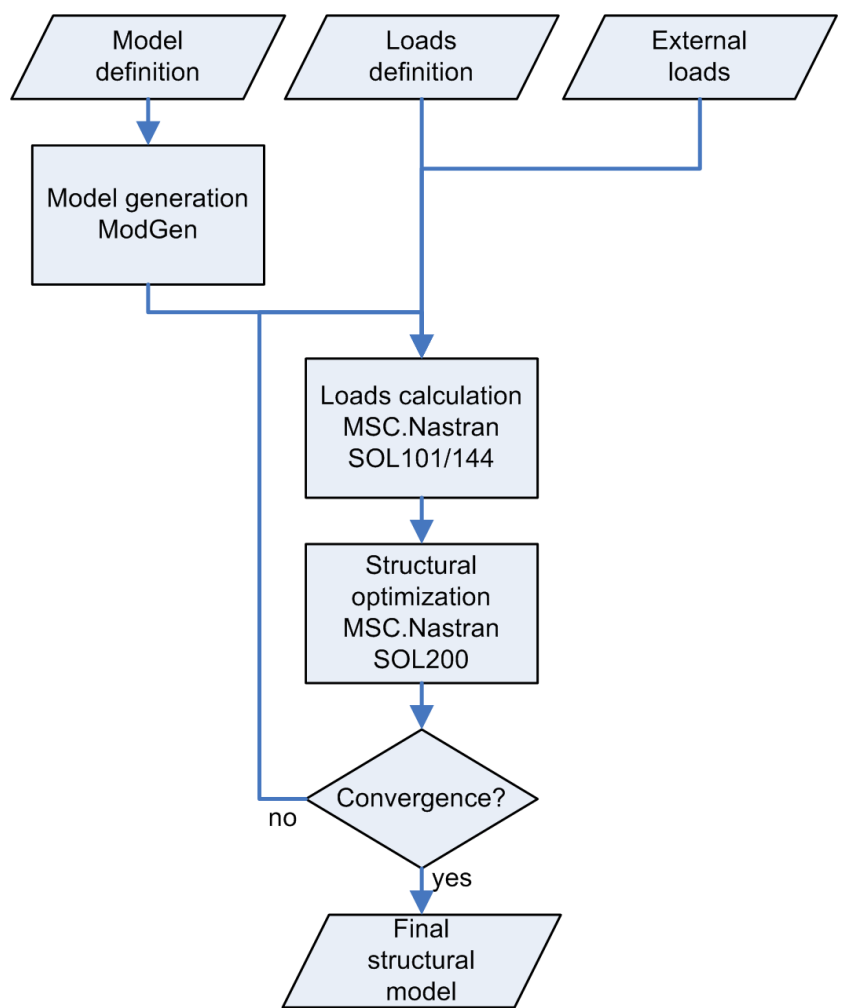

Fig. 2 Principal steps of the MONA process used for the DLR-F19

changed easily and results evaluated more quickly. As mentioned in the introduction, the geometrical shape of the DLF-F19 is given and the structural layout stayed fix after the initial set up. The parametric capabilities of ModGen are used for parametric element properties. During the sizing described in section 4.2 for example the material thickness is altered.

Developed for classical aircraft configurations, not all modules of ModGen are needed for the DLR-F19 (e.g. engine, pylon and fuselage model) while others (e.g. wingbox and mass model) needed to be extended. For example, the rather complex structural topology of the DLR-F19 is a challenge for such an automated process. Therefore the aircraft is divided into three independent segments: the fuselage region from the center line to the kink, the wing section and the triangular outer wing. There are five spars in the fuselage section with different orientations that eventually merge into two spars to form the wing box in the wing section. At the outer wing, the two spars continue until they merge at the wing tip. Many sharp corners exist all over the aircraft leading to meshing challenges if the points and edges are not merged or blended properly. This requires very robust algorithms, which needed to be adapted several times to ensure a smooth model generation. In addition, new features were developed for the use of composite materials and to provide better support for full, asym- 

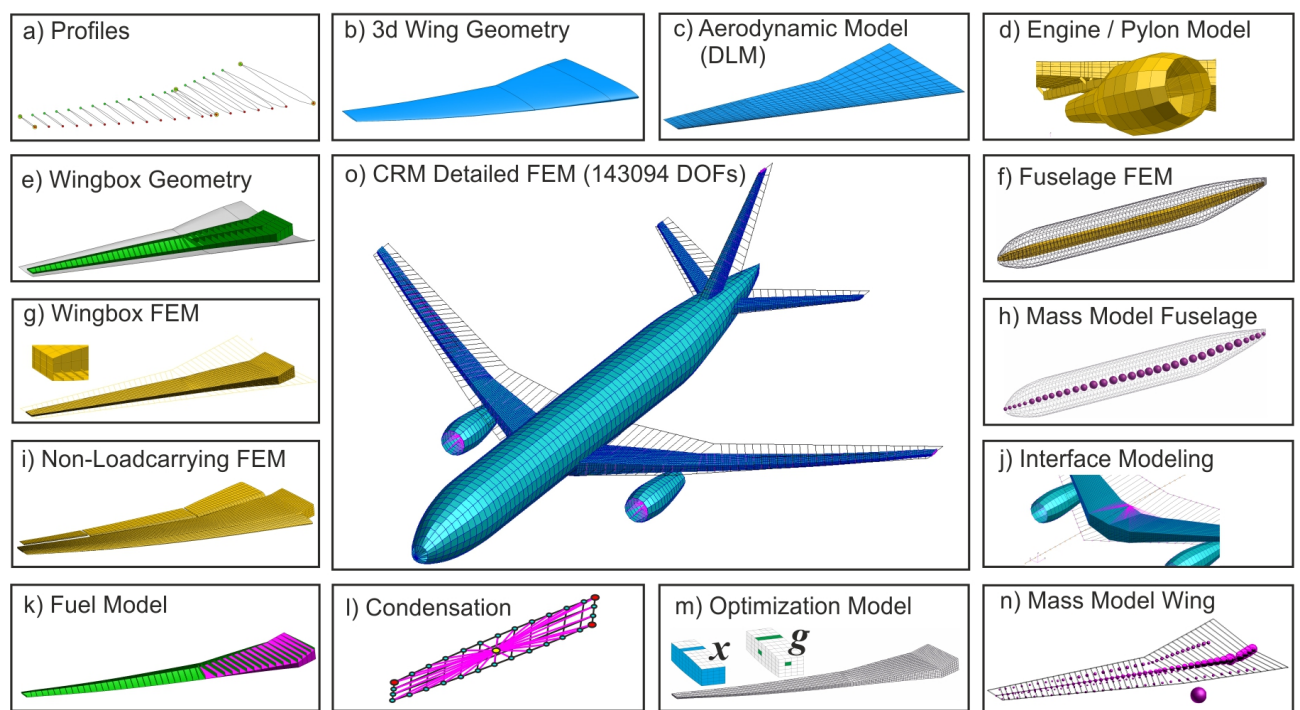

Fig. 1 Parametrization concept for simulation and optimization models of the FERMAT configuration [16]

metric models. While the structure is usually intended to be symmetric, both mass configuration and load condition could be asymmetric.

ModGen may be used as a stand-alone tool, as part of the ModGen/Nastran design process (MONA) [16, 19] or modules may be integrated in a fully automated design process [20]. The MONA process can be set up slightly differently, the principal steps used for the DLRF19 are depicted in Fig. 2. Once the model is generated with ModGen, MSC Nastran is started to calculate a set of load cases. Loads from external calculations (e.g. ground loads) may be considered at this step. With the resulting set of loads, a structural sizing is started, based on MSC Nastran's SOL200. These two steps form an iterative process that may be repeated until convergence regarding the optimum structural weight is achieved, resulting in a final structural model.

\section{Simulation Models of the DLR-F19}

\subsection{General Aspects}

The focus on aeroelastic aspects leads to a number of requirements which differ from a classical finite elements model for stress analysis. The structure should be as realistic as possible because global elastic characteristics such as wing bending and twist are of major interest. Local effects, like stress concentrations at sharp edges or at holes are neglected. This means that all primary structural components, such as spars, ribs, stringers and skin, should be modeled. In addition to the structural stiffness aspects, a mass model with properly distributed mass entities (e.g. structure, systems, payload, fuel) and the consideration of various mass

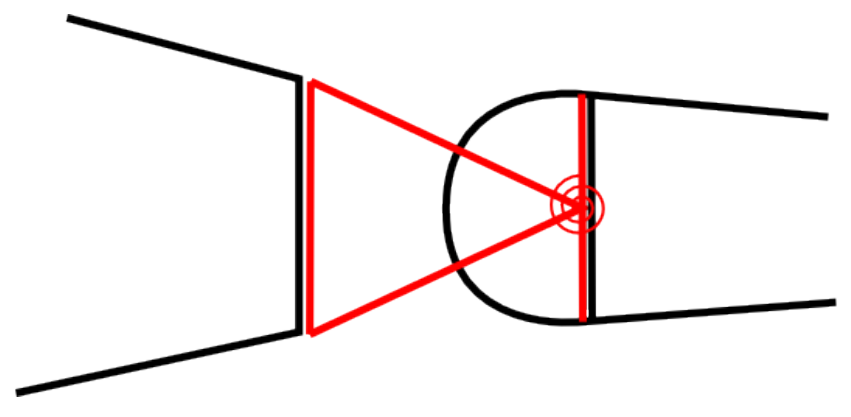

Fig. 4 Sketch of elastic control surface attachment to the wing

configurations (e.g. fuel, payload) are important to conduct proper dynamic calculations. The dynamic analysis of the stiffness and mass model should result in almost only global modes for a specified frequency range. Local modes are to be avoided.

As mentioned before, the geometry is based on a given configuration which also served for (scaled) wind tunnel models. The DLR-F19 has a half span of $7.68 \mathrm{~m}$ and an area of $77 \mathrm{~m}^{2}$. In a conceptual design process, Liersch and Huber [20] investigated the configuration, established a feasible design and a structural layout.

\subsection{Finite Element Model}

With this base, a finite element (FE) model consisting of 8054 GRID points and 8578 CQUAD4 and 6326 CBAR elements is created, as shown in Fig. 3. A righthand side and a corresponding symmetric left-hand side FE model are joined at the center line through RBE2 elements. The spars, ribs and skins are modeled as shell elements and are equipped with stiffening elements to 


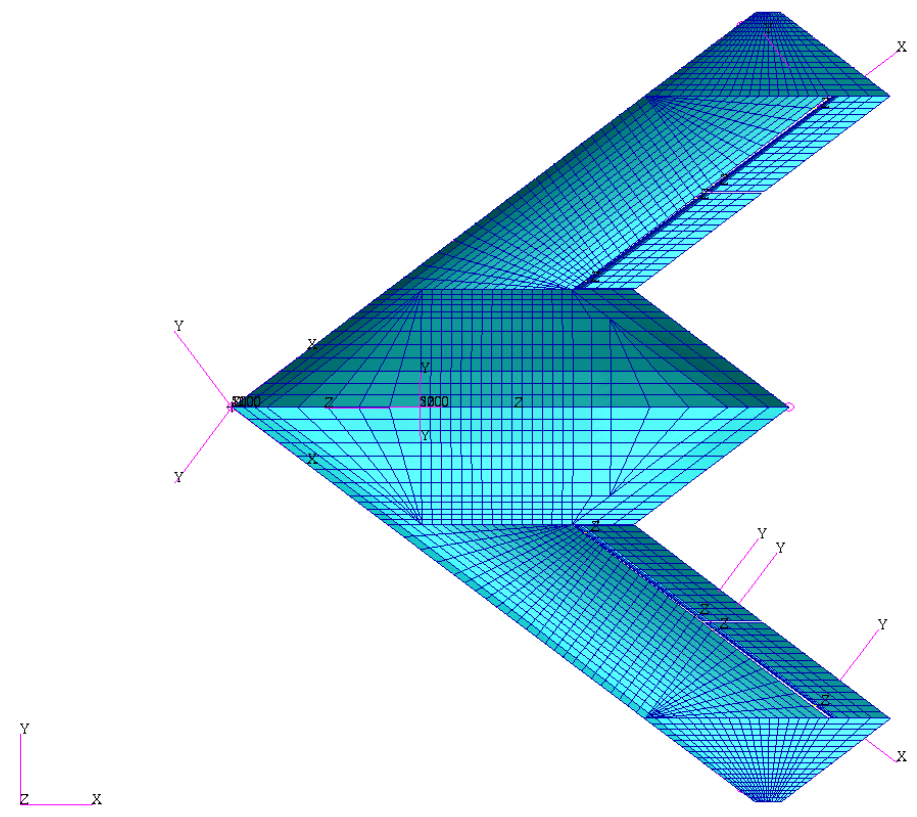

Fig. 3 Top view of structural model with local coordinate systems for laminate orientation and the hinge lines of the control surfaces

keep the buckling fields sufficiently small and reduce local eigenmodes. For the stringers, a hat profile is selected. The control surfaces are structurally modeled as well and attached to the wing elastically. The hinge concept is shown in Fig. 4 and consists of two parts. The first part, a triangular beam construction, is mounted on the wing's trailing edge. The second part is a vertical beam at the control surface's first spar, acting as reinforcement. Both parts are designed in such a way that the two hinge GRID points have exactly the same coordinates. Introducing a torsional spring element, stiffness about the rotation axis defined by a local coordinate system can be placed between these two points while all other degrees of freedom are fixed. The attachment stiffness can be controlled via material properties and the spring's stiffness. This adequate modeling ensures a realistic behavior and allows physically meaningful investigations on control surface loads.

For all structural components, suitable carbon fiber composite properties are chosen (see Table 1). For the skin, the $0^{\circ}$ plies are aligned along the leading edge using a local coordinate system to define the orientation. Material properties for unidirectional layers are provided by the DLR Institute of Composite Structures and Adaptive Systems. The material properties of the complete laminate setup are calculated as described in [25]. Using the stiffness matrix $\bar{K}_{i j}$ of each layer, the members $A_{i j}$ of the stiffness matrix $A$ of the complete laminate setup are calculated as stated in equation 1 . The so-called engineering constants for tensile elasticity $\hat{E}_{x}, \hat{E}_{y}$ and shear $G_{x y}$ are calculated from stiffness matrix $A_{i j}$ and the material thickness $t$ according to equations 2 .

$$
\begin{aligned}
\frac{A_{i j}}{t} & =\sum_{k=1}^{n} \frac{\bar{K}_{i j, k} \cdot t_{k}}{t} \\
\hat{E}_{x} & =\frac{1}{\left(A^{-1}\right)_{11} \cdot t} \\
\hat{E}_{y} & =\frac{1}{\left(A^{-1}\right)_{22} \cdot t} \\
G_{x y} & =\frac{1}{\left(A^{-1}\right)_{66} \cdot t}
\end{aligned}
$$

In order to reduce the size of the structural model, a condensation is often used for loads analysis tasks. In addition to the reduced computational costs, the model is also cleaned of modeling shortcomings and undesired effects, such as unrealistic local deformations due to high nodal loads. However, for flying wing configurations, such a condensation is not as straightforward as for classical configurations. The use of a loads reference axis is a good approach in the case of slender components like a classical aircraft wing, but appears to be 
Table 1 Overview of Chosen Carbon Fiber Laminates

\begin{tabular}{ll}
\hline components & laminate setup volume share $\left[0^{\circ} / \pm 45^{\circ} / 90^{\circ}\right]$ \\
\hline Skin & $40 / 40 / 20$ \\
Spars & $10 / 80 / 10$ \\
Ribs & $10 / 50 / 40$ \\
Stringer & $60 / 20 / 20$ \\
\hline
\end{tabular}

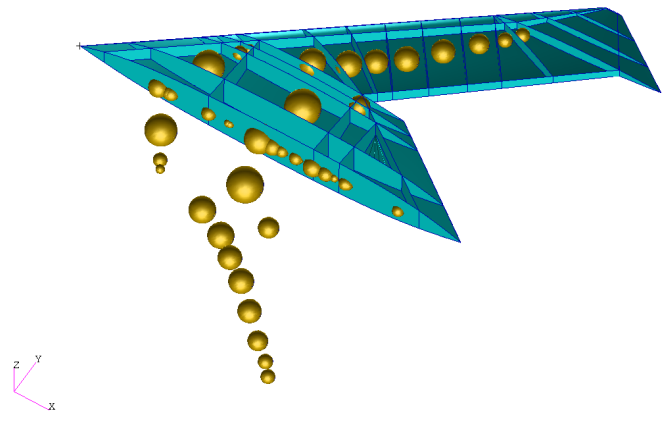

Fig. 5 Overview of Mass Model

less suitable in the case of a compact, non-slender configuration such as the DLR-F19. Based on these considerations, it was decided to stay with the full model and to accept possible disadvantages.

\subsection{Mass Model}

Proper mass modeling and the consideration of various mass configurations are important for an aeroelastic model. Due to the material density and dimensions, the structural mass is already included in the FE model. All non-structural masses are added in a separate mass model as so-called condensed or lumped masses, depicted in Fig. 5. The individual mass items are connected to the surrounding structure using RBE3 elements. From the huge amount of possible mass combinations, five distinct mass configurations are selected, shown in Table 2. Neither the payload nor the fuel level significantly change the center of gravity.

\subsection{Aerodynamic Model}

The classical approach with MSC Nastran's Doublet Lattice Method (DLM) [30] is chosen for the aerodynamics. The panels are arranged in such a way that 20 panels are defined in the flow direction, leading to a total of 840 panels, shown in Fig. 6. This discretization is sufficient for static applications but might need

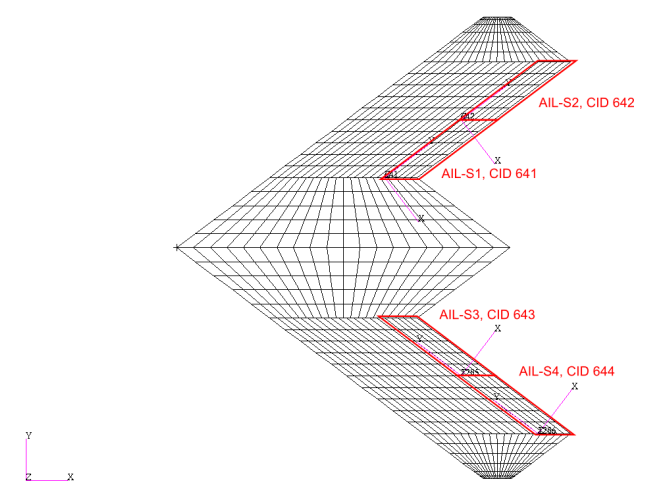

Fig. 6 Aerodynamic Mesh and Control Surfaces

to be refined to capture unsteady effects in dynamic calculations. To ensure that the panels are trapezoidal, the pointed wing tip is covered with aerodynamic panels only up to $90 \%$. The DLM is based on a matrix of aerodynamic influence coefficients (AIC), which depends on the Mach number $M a$ and reduced frequency $k$, with $k=0$ for the static case. The AIC matrix then relates an induced downwash $w_{j}$ on each aerodynamic panel to a pressure coefficient $c p$ as stated in equation 3.

$\{\Delta c p\}=A I C(M a, k) \cdot\left\{w_{j}\right\}$

The four control surfaces (AIL-S1, AIL-S2, AIL-S3, AIL-S4, shown in Fig. 6) each consist of $5 \times 5$ panels and their deflection is modeled by changing the induced downwash due to the rotation about their hinge lines.

\subsection{Coupling Strategies}

For the coupling of aerodynamic forces with the aircraft structure, a transformation matrix $T_{k g}$ is defined, which relates displacements of the structure $u_{g}$ to displacements of the aerodynamic grid $u_{k}$, see equation 4 . In addition, as in equation 5 , the transposed matrix $T_{k g}^{T}$ transforms forces and moments from the aerodynamic grid to the structure.

$\left\{u_{k}\right\}=T_{k g} \cdot\left\{u_{g}\right\}$ 
Table 2 Selected Mass Configurations

\begin{tabular}{lllll}
\hline Key & Description & Mass $[\mathrm{kg}]$ & $C G_{x}[\mathrm{~m}]$ & MAC [\%] \\
\hline - & structure only, no systems & 2756 & 6.78 & 48 \\
M1 & no fuel, no payload & 7361 & 5.72 & 30 \\
M2 & max. fuel, no payload & 12509 & 5.63 & 28 \\
M3 & max. fuel, max. payload & 14509 & 5.60 & 28 \\
M4 & no fuel, max. payload & 9361 & 5.65 & 29 \\
M5 & half fuel, max. payload & 11941 & 5.61 & 29 \\
\hline
\end{tabular}

$$
\left\{F_{g}\right\}=T_{k g}^{T} \cdot\left\{F_{k}\right\}
$$

There are many possibilities available to create the transformation matrix $T_{k g}$. One common option is to use a rigid body spline that relates every aerodynamic panel to the closest GRID point on the structure. However, this may result in high nodal forces when using a rather fine structural model. Therefore, surface splines are used, which distribute the aerodynamic forces more evenly to the structural points. To create such a surface spline for coupling, it is preferential to use only a subset of structural points on one side of the aircraft. In contrast to CFD (computational fluid dynamic) methods like the DLR Tau code [26], the DLM provides only a $\Delta c_{p}$ between the upper and lower side. For the spline, the Harder-Desmarais Infinite Plate Spline (IPS) [12] is selected, which is implemented in MSC Nastran's surface spline SPLINE1. The IPS delivers robust results in terms of smooth force distribution.

Using a single surface spline for the whole aircraft creates challenges, for example at the control surfaces. Aerodynamic forces on the control surface should act on the structure of the control surface only. This is difficult with a spline that somehow blurs forces and moments and would require component-wise splining. Due to the application of an interpolation method and the use of different discretizations (structure and aerodynamic), a similar phenomenon occurs when integrating the forces and moments of the structural points to calculate section forces. The structural points can be defined clearly, but the aerodynamic forces acting on them are subject of an interpolation. This might lead to a small imprecision. However, the interpolation employed in this paper is consistent.

\section{Sizing}

\subsection{Load Cases and Design Speeds}

For the sizing of the components of the structural model, load cases from three different areas of interest are considered:

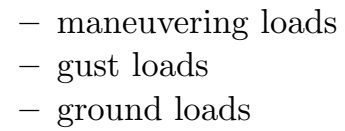

Normally, many different types of maneuvers are to be considered, such as pull-up, pull-down, roll or yaw. Due to insufficient control about the vertical axis, only symmetrical maneuvers are considered, e.g. $1 \mathrm{~g}$ horizontal flight, $2.5 \mathrm{~g}$ pull-up and $-1.0 \mathrm{~g}$ push-down. For dive cases at dive speed $V_{d} / M_{d}$, the push-down is reduced to $0.0 \mathrm{~g}$. The applied set of maneuvers is in close relation to the maneuvers required by the certification specifications CS-25 for large transportation aeroplanes [10]. During a collaboration between DLR and Airbus Defence and Space as part of the Mephisto project, the loads department defined a set of additional aircraft response parameters for use in the preliminary design. These response parameters are representative for flight conditions that an aircraft of this type might encounter during a typical mission.

The gust loads are calculated according to the certification specifications CS 23.341 for normal aircraft [9]. Using the Pratt formula given in equation 6, gusts are converted to an equivalent load factor $n_{z}$. The loads are then calculated as static maneuvers. More detailed information can be found in the NACA report 1206 [24].

$$
\begin{aligned}
n_{z} & =1.0 \pm \frac{k_{g} \cdot \rho_{0} \cdot U_{d e} \cdot V \cdot\left(\frac{d C l}{d \alpha}\right)}{2 \cdot\left(\frac{W}{S}\right)} \text { with }: \\
k_{g} & =\text { gust alleviation factor } \\
\rho_{0} & =\text { density of air at sea level } \\
U_{d e} & =\text { derived gust velocities } \\
V & =\text { equivalent air speed } \\
\frac{d C l}{d \alpha} & =\text { lift curve slope } \\
\frac{W}{S} & =\text { wing loading }
\end{aligned}
$$

These load cases are calculated for all five mass configurations (see Table 2), for the design cruise and design dive speeds $V_{c} / M_{c}$ and $V_{d} / M_{d}$ and at various Flight Levels $(\mathrm{FL}=$ altitude $[\mathrm{ft}] / 100)$, shown in Fig. 7. The design cruise speed is Mach 0.8 at sea level and increases 


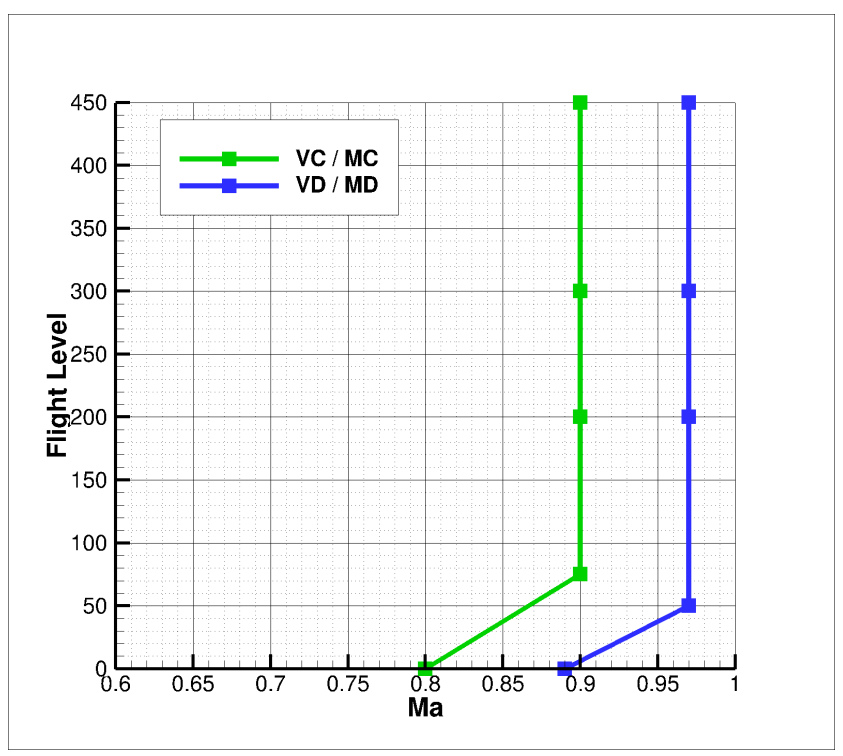

Fig. $7 V_{c} / M_{c}$ and $V_{d} / M_{d}$ border and flight points for the considered load cases

to Mach 0.9 at FL 75 while the design dive speed is Mach 0.9 at sea level and increases to Mach 0.97 at FL 55. Finally, landing loads are calculated using the DLR in-house tool LGDesign based on analytical formula and handbook methods. The maximum dynamic attachment loads of the landing gear are introduced into the structure as an additional load case. All in all, 216 load cases are taken into account.

\subsection{Optimization Model}

The objective is to minimize the structural weight while keeping the responses such as element strains inside their boundaries. The task is treated as a mathematical optimization problem and is formally defined in equation 7 . Therein, $f$ is the objective function with vector $x$ containing the design variables and $g$ is the constraint vector.

$\operatorname{Min}\left\{f(x) \mid g(x) \leq 0 ; x_{\text {lower }} \leq x \leq x_{\text {upper }}\right\}$

With $1200 \mu \mathrm{m} / \mathrm{m}(0.12 \%)$ allowable compression and $1500 \mu \mathrm{m} / \mathrm{m}(0.15 \%)$ allowable tension, derived from the material properties for unidirectional layers, the boundaries are rather conservative and provide a margin for possible subsequent changes in the configuration. Currently, the material thickness of the upper and lower skin, spars and ribs are defined as optimization variables. The elements are grouped in areas and linked in order to define one variable per area. The element with the highest strains therefore governs the whole area. The structural model is designed in a way that the left and right side have the same properties, whereby the
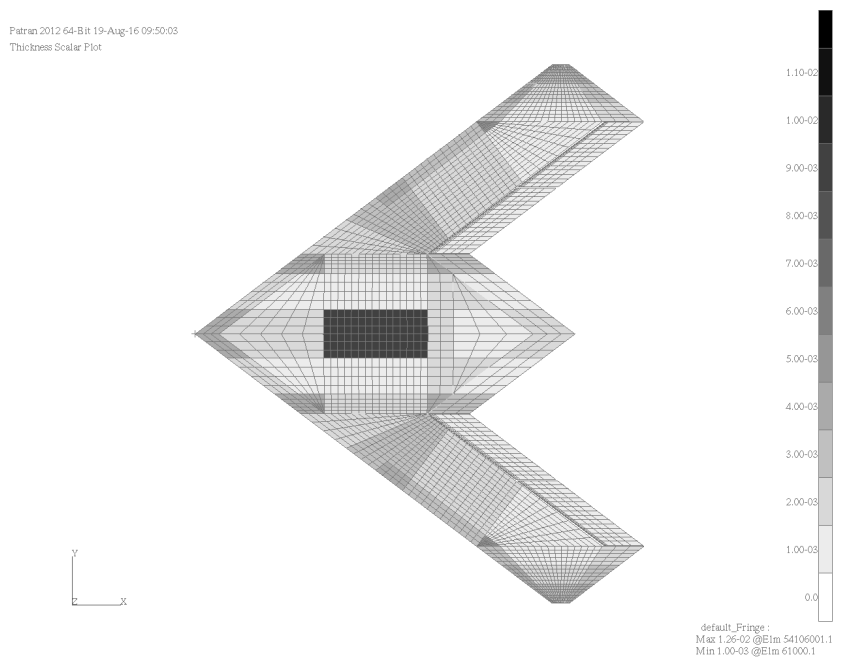

Fig. 8 Element thickness, after optimization

corresponding design areas are changed simultaneously as well. This is to ensure symmetry, a necessity when asymmetrical load cases are considered. Summing up, the optimization problem has 168 design variables. The 6758 design responses multiplied by 216 load cases lead to $\sim 1.5$ million constraints.

It turns out that the optimization converges rather quickly and only two or three loops are necessary. However, experience has shown that there is no clear convergence in the sense of a function developing towards a relative change of the objective function $f$ less than 0.001 , the default value for MSC Nastran SOL200. Instead, the results vary in a range of $\pm 100 \mathrm{~kg}$. This is acceptable for such kind of optimization and makes up only $2-3 \%$ of the final weight of the pure structure. Starting with an initial setup (uniform material thickness), the pure structural weight dropped to a final weight of 2750 $\mathrm{kg}$. The final element thickness distribution is displayed in Fig. 8. One can see a thickness variation starting at the minimum material thickness of $1.25 \mathrm{~mm}$ (light grey) up to $9.28 \mathrm{~mm}$ (dark grey) in the center region where many systems and the engine is installed. The resulting strain distribution is displayed for one load case in Fig. 9. After the optimization, they reach the upper limit of $1500 \mu \mathrm{m} / \mathrm{m}$. In addition, they are distributed evenly among the skin elements, suggesting a efficient use of the material available.

\section{Loads Analysis}

\subsection{General Aspects}

The resulting structural model is used to conduct a comprehensive loads analysis campaign. The load cases and calculations are basically the same as for the siz- 


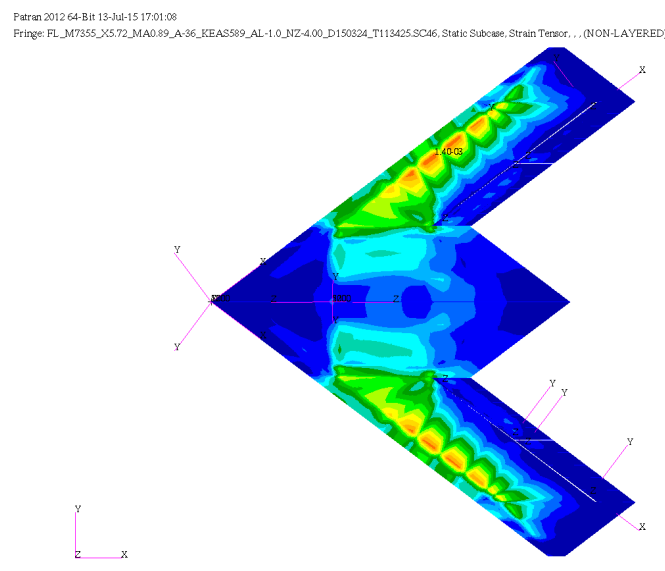

Fig. 9 Element strain, after optimization

ing. While nodal loads were required for the sizing, now the emphasis lies on so-called interesting quantities. Interesting quantities usually include cutting forces and moments at various stations (e.g. along the wing or fuselage) and attachment loads (e.g. from control surfaces, payload, landing gear, etc.). These quantities are extracted with the help of monitoring stations. The monitoring stations used in this work are taken from a collaboration with the Airbus Defence \& Space loads department. Difficulties for a configuration like the DLR-F19 consist in finding clear cuts of the structure and avoiding overdetermined regions due to unknown load paths, e.g. at the control surface hinges or in the payload bay. The choice of proper coordinate systems is also important. A local coordinate system with its $y$-axis along the leading edge provides more physically meaningful and understandable section forces and moments for the wing than a global coordinate system. However, with cuts in the flow direction, a small "negative" area occurs that counteracts for example the moment about the $x$-axis (see sketch of MON3 included in Fig. 10 ). The equations of motion solved for the static maneuver calculation are given in equation 8 . The stiffness matrix $K$ and the mass matrix $M$ are multiplied by flexible deformations and rigid body motions $u$ as well as accelerations $\ddot{u}$. Aerodynamic forces due to structural deformations are introduced by the matrix $Q$. Finally, they are related to a vector of applied forces and moments $P$. The calculation is usually conducted with the reduced structural degrees of freedom (a-set in MSC Nastran [21]). As mentioned in section 3.2, in this case a condensation to a load reference axis is not desired, so the a-set is equivalent to the full model.

$\left(K_{a a}-q_{\infty} \cdot Q_{a a}(M a)\right) \cdot\left\{u_{a}\right\}+M_{a a} \cdot\left\{\ddot{u}_{a}\right\}=\left\{P_{a}\right\}$

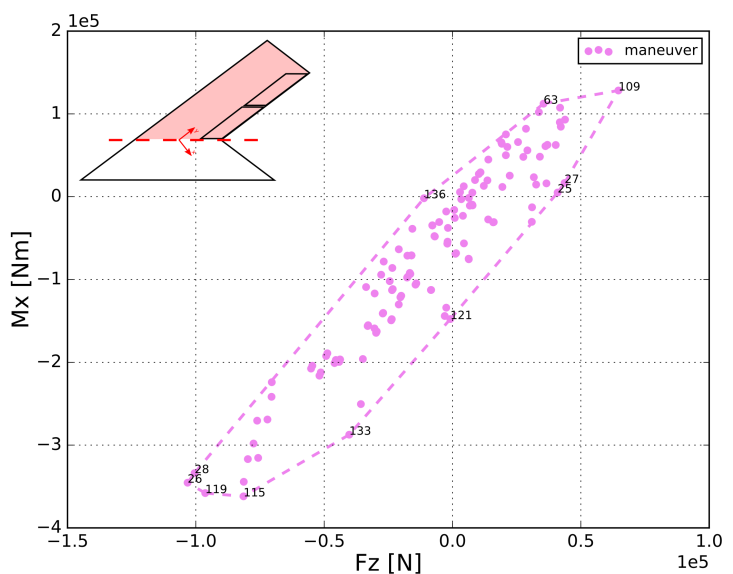

Fig. 10 Cutting forces $F_{z}$ and moments $M_{x}$ at the wing root (MON3) for maneuver loads

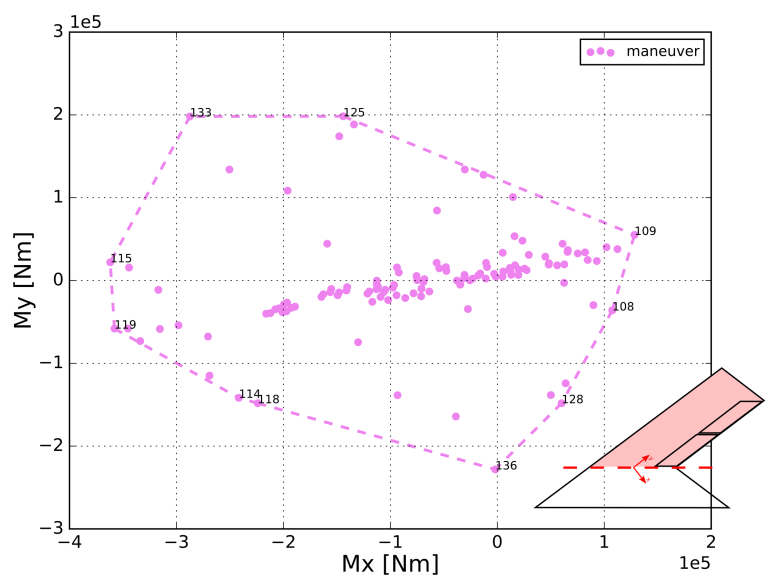

Fig. 11 Cutting moments $M_{x}$ and $M_{y}$ at the wing root (MON3) for maneuver loads

The underlying equation of motion for dynamic gust calculation is given in equation 9 and differs from the static one. First, it is performed in modal coordinates $h$. In addition, matrix $Q$ now depends on Mach number $M a$ and reduced frequency $k$ and includes unsteady aerodynamic effects. The aerodynamic loads due to a discrete 1-cosine gust shapes are applied via $P_{h}(\omega)$.

$$
\begin{array}{r}
\left(-M_{h h} \omega^{2}+K_{h h}-q_{\infty} \cdot Q_{h h}(M a, k)\right) \cdot\left\{u_{h}\right\} \\
=\left\{P_{h}(\omega)\right\}
\end{array}
$$

\subsection{Maneuvering Loads}

In Fig. 10 and 11 the three major cutting loads $F_{z}, M_{x}$ and $M_{y}$ due to maneuver load cases are plotted for the wing root. They are plotted as two-dimensional load envelopes as far as combinations of the cutting loads lead 


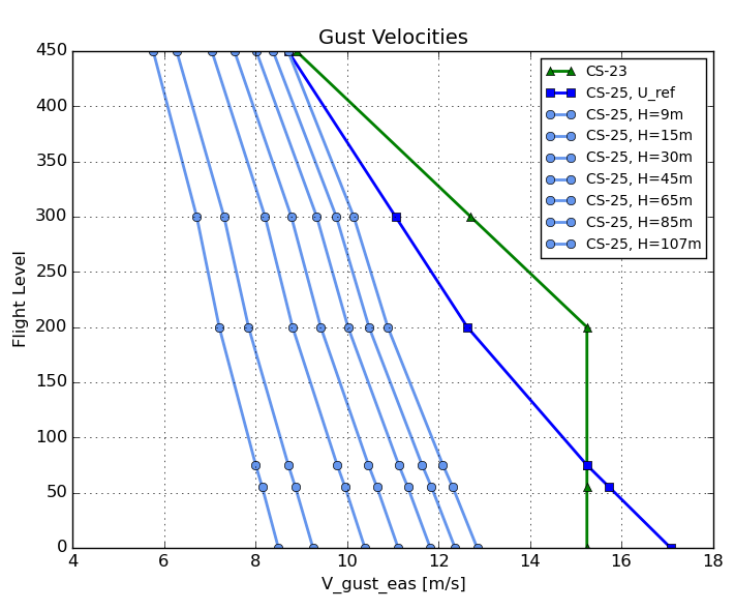

Fig. 12 Assumed gust velocity profiles for Pratt and 1-cosine gusts

to the maximum strains. In Fig. 10 one can see that $F_{z}$ has its highest amplitude in the negative region. This has several reasons. First, the wing is twisted negatively towards the outer regions. Secondly, for a positive angle of attack, the control surfaces deflect upwards to compensate the pitching moment. This upward deflection reduces the lift on the wing even further. Hence, for most horizontal flight conditions only the fuselage generates positive lift while the wing generates negative lift. The sign and amplitude of $M_{y}$ changes significantly with the maneuvers. Both high load factors $N_{z}$ and roll rates/accelerations lead to large control surface deflections which cause a negative moment about the $\mathrm{y}$-axis for upward and a positive moment for downward deflections. As expected, the highest maneuver loads are caused by the design maneuvers which combine high load factors $\left(N_{z}\right.$ between $-1.8 \mathrm{~g}$ and $\left.+4.5 \mathrm{~g}\right)$ with high roll rates / accelerations. The maneuvers calculated according to CS 25.337 have lower load factors of $-1.0 \mathrm{~g}$ and $2.5 \mathrm{~g}$, thus showing lower loads.

\subsection{Gust Loads}

For the sizing of the DLR-F19 structure, the Pratt formula was applied to account for gust loads. Now, a dynamic gust analysis is performed according to the certification specifications CS 25.341 for large aircraft [10]. The aircraft is exposed to a series of vertical 1-cosine shaped gusts with lengths $H$ of $9,15,30,45,65,85$ and $107 \mathrm{~m}$, both positive and negative and at the same altitudes, speeds and with the same mass configurations as before. Finally, the results are superposed with loads of a $1.0 \mathrm{~g}$ horizontal level flight. For the Pratt formula, both wing area and aircraft weight are input parameters. As the wing area is rather large in comparison to

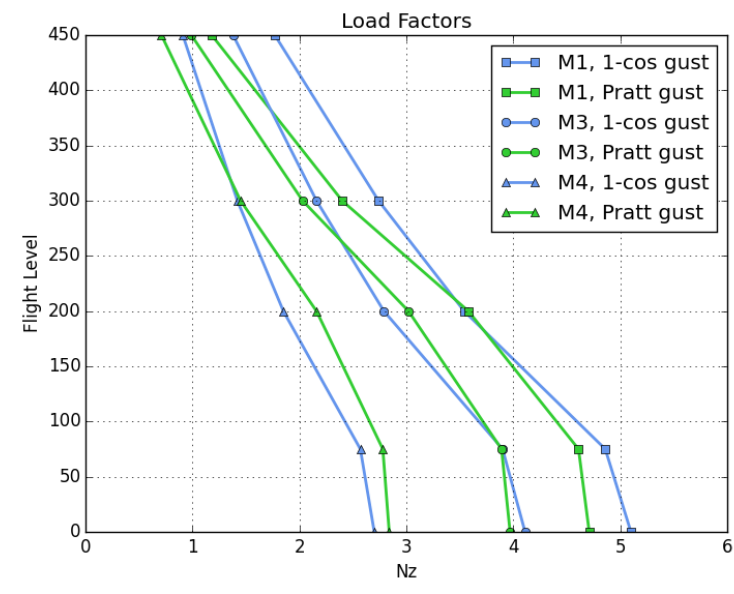

Fig. 13 Load factors $N_{z}$ obtained from Pratt and 1-cosine calculations

the aircraft weight, the highest calculated load factors are $1.0 \mathrm{~g} \pm 4.7 \mathrm{~g}$ (at sea level, lightest mass configuration M1). First, one can compare the resulting load factors from the dynamic 1-cosine simulation by summing up all inertia forces and dividing by the aircraft weight. It turns out that the dynamic 1-cosine simulation does not necessarily produce smaller load factors, but in general they are lower at lower altitudes and higher at higher altitudes. This can be explained by looking at the underlying gust velocities shown in Fig. 12. The green curve is derived from CS 23.333 and used for the Pratt formula. The dark blue curve shows the reference gust velocities derived from CS 25.341. These reference gust velocities are further reduced by a flight profile alleviation factor and adapted to the gust length $H$, resulting in individual gust velocities for each gust length. In general, the gust velocities used for the Pratt formula are higher than those of the 1-cosine gust, with a peak at FL 200. With increasing altitude, the difference gets smaller. This trend is also reflected in the cutting forces. For academic purposes, one might correct these differences by simply using the same gust velocities for both types of calculations, as in [11].

A second observation is the tendency of Pratt load factors to be higher with increasing aircraft mass as compared to 1-cosine gusts. In Fig. 13 load factors $N_{z}$ are plotted for both Pratt and 1-cosine gusts for mass configurations M1, M3 and M4. Again, one can see a peak for Pratt load factors at Flight Level 200. More important, for the lightest configuration M1, Pratt load factors are lower than those obtained from 1-cosine calculations. This is no longer the case for the heaviest configuration M4. Actually, this tendency is already discovered for mass configuration M3, where only a payload of two tons is added in comparison to M1. The reason 


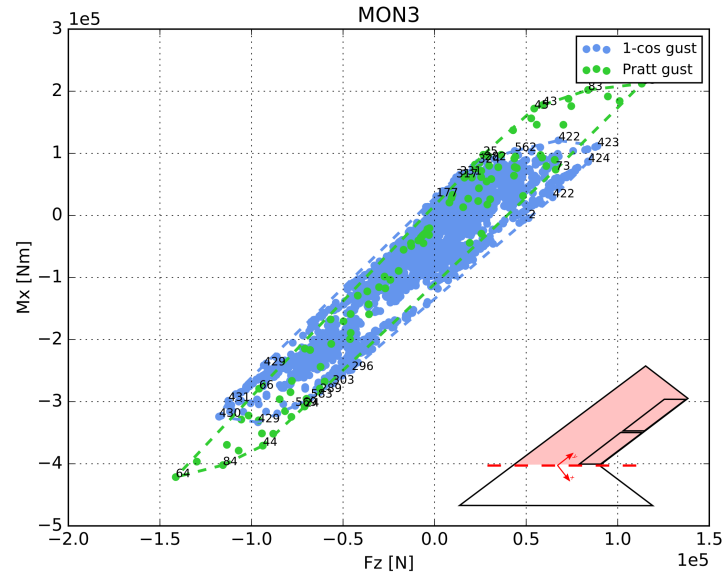

Fig. 14 Cutting forces $F_{z}$ and moments $M_{x}$ at the wing root (MON3) for Pratt and 1-cosine gust loads

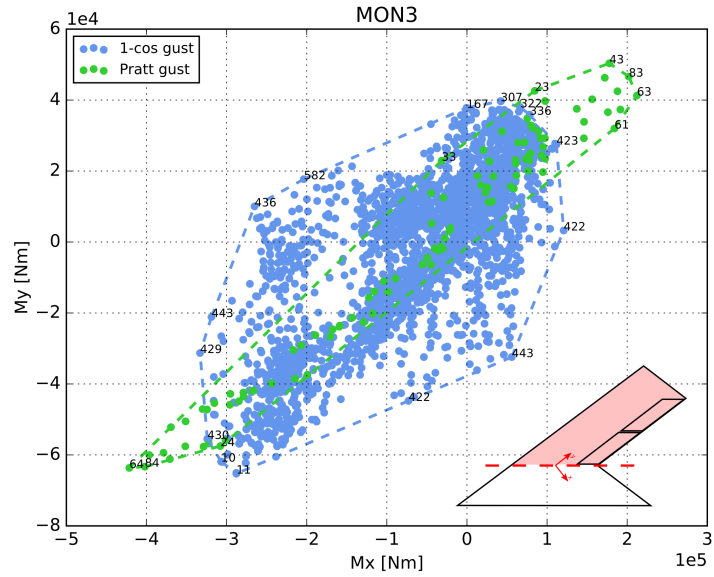

Fig. 15 Cutting moments $M_{x}$ and $M_{y}$ at the wing root (MON3) for Pratt and 1-cosine gust loads

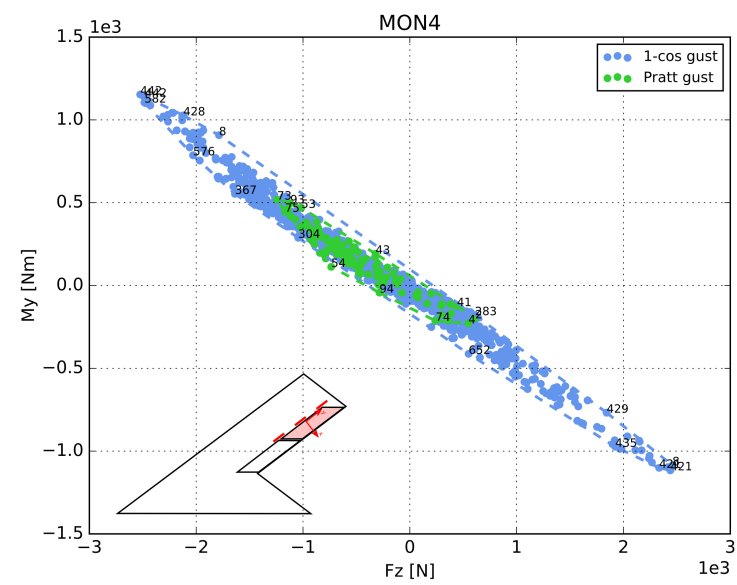

Fig. 16 Hinge forces $F_{z}$ and moments $M_{y}$ of the outer control surface (MON4) for Pratt and 1-cosine gust loads for this effect could be explained by the gust alleviation factor $k_{g}$ used in the Pratt formula, which might not be suitable for "flying wings" or aircraft with highly swept wings. The effect is represented in the loads plot in Fig. 14 as well. Cutting forces $F_{z}$ and moments $M_{x}$ are plotted for a monitoring station at the wing root. Fig. 14 shows that the loads in green obtained from Pratt are higher than those from the 1-cosine gust in blue. In fact, the highest cutting forces are produced by mass configuration M3. Looking at the cutting moments $M_{x}$ and $M_{y}$ in Fig. 15, the Pratt loads are again higher than those from 1-cosine calculations. However, the area covered by the blue dots is much larger than the green area, indicating that higher torsional moments occur when performing a 1-cosine simulation. An explanation for this behavior is that the 1-cosine simulations include structural dynamics while the Pratt calculations are quasi-static. This has a significant impact on the control surfaces, for example, which are attached elastically to the wing as described in section 3.2. A close look at the hinge forces and moment of the outer control surface in Fig. 16 reveals that the dynamic simulations lead to much higher hinge forces and moments than the quasi-static ones. While longer gust are more similar to the forces and moments obtained from a quasi-static Pratt calculation, the extitation due to shortes gusts is much stronger. Due to the elastic attachment of the control surfaces to the wing's trailing edge, the excitation with to short gusts causes inertia forces, which are very high. In addition, once the aerodynamic loads fade away, the control surface returns into its initial position. The overshoot that occurs during that process actually produces the loads of highest amplitude. This dynamic behaviour is not captured properly by a quasistatic Pratt calculation. In addition, one might apply correction factors to the control surface aerodynamics to conpensate effects of thickness and viscosity, which are not captured by the DLM. Various correction methods are summarized in [23]. This correction would apply to both Pratt and 1-cosine simulations. The loads might be lower, but the relative difference should remain the same.

\section{Conclusion and Outlook}

In this paper, the DLR's parametric modeling process MONA is used successfully to create an aeroelastic model for a "flying wing" configuration. The resulting model is sized for 216 load cases including maneuver, gust and landing loads. The structural model is comparatively detailed for a conceptual phase and a model condensation typical for loads analysis is avoided. Together with 
a surface spline, this is a more physical way of calculating aircraft loads and introducing them to the structure that doesn't require any loads reference axes. Also, new materials are employed, since the aircraft is fabricated completely out of carbon fiber composites. Once the model is sized, a comprehensive loads campaign is conducted. In addition to maneuver loads, gust loads are calculated and compared using both the quasi-static Pratt formula and the dynamic 1-cosine gust. Differences can be explained by the various underlying gust velocities and by the gust alleviation factor $k_{g}$. However, accounting for gust loads using the Pratt formula is a reasonable method with less compuational effort for a parametric modeling process that takes place in a conceptual phase. It might produce higher loads, but leaves a margin for a comprehensive loads analysis campaign if the aircraft is expected to be certified according to CS-25.

In the future, aero-structural coupling could be improved by component-wise splining. Loads integration could be done by integrating aerodynamic forces directly, without splining them to the structure. This presumably results in more accuracy. The modeling of carbon fiber composites could also be enhanced by modeling all individual plies instead of calculating engineering constants for the whole laminate setup. Doing that, one could also consider the failure characteristics of carbon fiber composites in a more sophisticated manner and use them as constraints for the structural sizing. A lot of work was put into determining the center of gravity for the different mass configurations. However, the position of the center of pressure is rather vague, as the DLM is only valid for subsonic speeds. Shifting the center of pressure might change the trim results and therefore the loads could change significantly as well. In addition, the aerodynamic flow is expected to have very three dimensional characteristics with a pronounced cross-flow in spanwise direction, which is not captured properly by the DLM. That topic is investigated more deeply in [29], where an enhancement of the DLM aerodynamics with more sophisticated results obtained from CFD and its impact on maneuver loads is presented.

Acknowledgements The authors wish to thank Georg Wellmer and Sara Kirchmayr from the Airbus Defence \& Space loads department for the prolific collaboration.

\section{References}

1. Boeing Phantom Works to Lead Research on X-48B Blended Wing Body Concept. http: //boeing.mediaroom.com/2006-05-04-Boeing-PhantomWorks-to-Lead-Research-on-X-48B-Blended-WingBody-Concept (2006)
2. X-48B BWB Team Completes Phase 1 Test Flights. http://www.nasa.gov/centers/dryden/news/ NewsReleases/2010/10-12.html (2013)

3. X-47B UCAS Unmanned Combat Air System Data Sheet. http://www.northropgrumman.com/capabilities/ x47bucas/documents/ucas-d_data_sheet.pdf (2014)

4. X-47B UCAS Makes Aviation History... Again! http://www.northropgrumman.com/Capabilities/ X47BUCAS/Pages/default.aspx (2015)

5. Britt, R.T., Jacobson, S.B., Arthurs, T.D.: Aeroservoelastic Analysis of the B-2 Bomber. Journal of Aircraft 37(5), 745-752 (2000). DOI 10.2514/2.2674

6. Chen, D., Britt, R., Roughen, K., Stuewe, D.: Practical Application of Multidisciplinary Optimization to Structural Design of Next Generation Supersonic Transport. In: 13th AIAA/ISSMO Multidisciplinary Analysis Optimization Conference. American Institute of Aeronautics and Astronautics, Fort Worth, Texas (13-15 September 2010). DOI $10.2514 / 6.2010-9311$

7. De La Garza, McCulley, Johnson, Hunten, Action, Skillen, Zink: Recent Advances in Rapid Airframe Modeling at Lockheed Martin Aeronautics Company. In: RTOMP-AVT-173 Workshop. North Atlantic Treaty Organization, Bulgaria (16-19 May 2011)

8. Deck, U., Schwochow, J.: Aeroelastische Voruntersuchung des Nurflügelsegelflugzeugs AK-X. In: Deutscher Luft- und Raumfahrtkongress 2015. Rostock (22-24 September 2015)

9. European Aviation Safety Agency (ed.): Certification Specifications for Normal, Utility, Aerobatic, and Commuter Category Aeroplanes CS-23. Amendment 3 (2012)

10. European Aviation Safety Agency (ed.): Certification Specifications for Large Aeroplanes CS-25. Amendment 16 (2015)

11. Handojo, V., Klimmek, T.: Böenlastanalyse der vorwärts gepfeilten ALLEGRA-Konfiguration. In: Deutscher Luftund Raumfahrtkongress 2015. Rostock (22-24 September 2015)

12. Harder, R.L., Desmarais, R.N.: Interpolation using surface splines. Journal of Aircraft 9(2), 189-191 (1972). DOI 10.2514/3.44330

13. Huber, K., Schütte, A., Rein, M.: Numerical Investigation of the Aerodynamic Properties of a Flying Wing Configuration. In: American Institute of Aeronautics and Astronautics (ed.) 32nd AIAA Applied Aerodynamics Conference. New Orleans, Louisiana (25-28 June 2012)

14. Huber, K.C., Vicroy, D.D., Schuette, A., Huebner, A.: UCAV model design and static experimental investigations to estimate control device effectiveness and Control capabilities. In: American Institute of Aeronautics and Astronautics (ed.) 32nd AIAA Applied Aerodynamics Conference. Atlanta, GA (2014). DOI 10.2514/6.20142002

15. Kelm, Läpple, Grabietz: Wing primary structure weight estimation of transport aircrafts in the pre-development phase. In: 54th Annual Conference of Society of Allied Weight Engineers, Inc. Huntsville, Alabama (22-24 May 1995)

16. Klimmek, T.: Parametric set-up of a structural model for fermat configuration for aeroelastic and loads analysis. Journal of Aeroelasticity and Structural Dynamics (2), 31-49 (2014). DOI 10.3293/asdj.2014.27

17. Klimmek, T.: Parameterization of topology and geometry for the multidisciplinary optimization of wing structures. In: CEAS 2009 - European Air and Space Conference. Manchester, United Kingdom (26-29 October 2009) 
18. Krüger, W., Cumnuantip, S., Liersch, C.M.: Multidisciplinary Conceptual Design of a UCAV Configuration. In: Proceedings AVT-MP173. Sofia, Bulgaria (16-18 May 2011)

19. Krüger, W., Klimmek, T., Liepelt, R., Schmidt, H., Waitz, S., Cumnuantip, S.: Design and aeroelastic assessment of a forward-swept wing aircraft. CEAS Aeronautical Journal 5(4), 419-433 (2014). DOI 10.1007/s13272014-0117-0

20. Liersch, C.M., Huber, K.C.: Conceptual Design and Aerodynamic Analyses of a Generic UCAV Configuration. In: American Institute of Aeronautics and Astronautics (ed.) 32nd AIAA Applied Aerodynamics Conference. Atlanta, GA (16-20 June 2014). DOI 10.2514/ 6.2014-2001

21. MSC.Software Corporation: Set Definition. In: D.M. McLean (ed.) MSC Nastran Linear Static Analysis User's Guide, vol. 2012.2, pp. 485-489 (2012)

22. Nangia, R., Palmer, M.: A Comparative Study of UCAV Type Wing Planforms - Aero Performance \& Stability Considerations. In: 23rd AIAA Applied Aerodynamics Conference. American Institute of Aeronautics and Astronautics, Toronta, Ontario, Canada (6-9 June 2005). DOI 10.2514/6.2005-5078

23. Palacios, R., Climent, H., Karlsson, A., Winzell, B.: Assessment of Strategies for Correcting Linear Unsteady Aerodynamics Using CFD or Test Results. In: International Forum on Aeroelasticity and Structural Dynamics (2001)

24. Pratt, K.G., Walker, W.G.: A Revised Gust-Load Formula and a Re-evaluation of V-G Data Taken on Civil Transport Airplanes From 1933 to 1950. Tech. Rep. Report 1206, NACA (1953)

25. Schürmann, H.: Konstruieren mit Faser-KunststoffVerbunden. Springer, Berlin; Heidelberg; New York (2007)

26. Schwamborn, D., Gerhold, T., Heinrich, R.: The DLR Tau-Code: Recent Applications in Research and Industry. In: ECCOMAS CDF 2006 (2006)

27. Schweiger, J., Sensburg, O., Berns, H.J.: Aeroelastic Problems and Structural Design of a Tailless CFCSailplane. In: Second International Symposium on Aeroelasticity and Structural Dynamics. Aachen (April 1-3 1985)

28. Tianyuan, H., Xiongqing, Y.: Aerodynamic/Stealthy/Structural Multidisciplinary Design Optimization of Unmanned Combat Air Vehicle. Chinese Journal of Aeronautics 22(4), 380-386 (2009). DOI 10.1016/S1000-9361(08)60114-4

29. Voss, A., Klimmek, T.: Maneuver Loads Calculation with Enhanced Aerodynamics for a UCAV Configuration. In: AIAA Modeling and Simulation Technologies Conference. American Institute of Aeronautics and Astronautics, Washington, D.C. (2016). DOI 10.2514/6.2016-3838

30. W. P. Rodden, Albano: A Doublet Lattice Method For Calculating Lift Distributions on Oscillation Surfaces in Subsonic Flows. In: N. Northorp (ed.) AIAA 6th Aerospace Sciences Meeting. New York (22-24 January 1968)

31. Wenzel, J., Sinapius, M., Gabbert, U.: Primary structure mass estimation in early phases of aircraft development using the finite element method. CEAS Aeronautical Journal 3(1), 35-44 (2012). DOI 10.1007/s13272-0110040-6

32. Wiggen, S., Voß, G.: Development of a wind tunnel experiment for vortex dominated flow at a pitching Lambda wing. CEAS Aeronautical Journal 5(4), 477-486 (2014). DOI 10.1007/s13272-014-0121-4

33. Winther, B.A., Hagemeyer, D.A., Britt, R.T., Roden, W.P.: Aeroelastic Effects on the B-2 Maneuver Response. Journal of Aircraft 32(4), 862-867 (1995). DOI 10.2514/ 3.46802

34. Woolvin, S.: A Conceptual Design Studies of the 1303 UCAV Configuration. In: 24th Applied Aerodynamics Conference. American Institute of Aeronautics and Astronautics, San Francisco, California (5-8 June 2006). DOI $10.2514 / 6.2006-2991$ 